\title{
Entre Itten y Gropius: algunas contradicciones culturales de la Bauhaus
}

\section{Between Itten and Gropius: Some Cultural Contradictions in the Bauhaus}

\author{
KUNDALINI MUÑOZ CERVERA AGUILAR \\ Facultad de Filosofía y Letras \\ UNIVERSIDAD NAGIONAL AUTÓNOMA DE MÉXICO | México \\ Contacto: kundalinimunoz@filos.unam.mx
}

\section{Resumen}

Una de las contradicciones más llamativas al interior de lo que se conoce como la escuela Bauhaus se dio entre el futurismo tecnicista-humanista de Walter Gropius y el ocultismo orientalista de otros miembros, como Johannes Itten. Aunque ambos buscaban armonizar la existencia del ser humano con el entorno que habita, las teorías en las que ambas tendencias basaban sus prácticas llegaban a ser irreconciliables. Era inconcebible que el ideal artístico de Itten estuviera al servicio de la humanidad en su conjunto cuando defendía nociones protoracistas sobre la capacidad exclusiva de un grupo étnico para alcanzar dicha armonía. Muchas de estas ideas quedaron incluso plasmadas en su obra de arte y no sólo en escritos y discursos publicados. El propio interés de Itten por la cultura y el arte oriental convivió incómodamente con estas tendencias, dejando un legado ambivalente y problemático, el cual ha sido recientemente señalado por algunos estudiosos del movimiento. Aquí analizaremos algunas aristas de esta contradicción y sus implicaciones desde el punto de vista de la comparatística. Resulta necesario plantearnos cómo se deben ver estas inconsistencias si queremos evitar caer en una postura trivial y poco crítica de un movimiento cultural tan relevante como lo fue la escuela Bauhaus.

Palabras clave: Bauhaus, Johannes Itten, Walter Gropius, ocultismo, Mazdaznan, comparatística

\begin{abstract}
One of the most striking contradictions within what is known as the Bauhaus school was the one that occurred between the technical humanist futurism of Walter Gropius and the orientalist occultism of other members, such as Johannes Itten. Although both sought to harmonize the existence of human beings with the environment they inhabit, the theories on which both trends based their practices were irreconcilable. It was inconceivable that Itten's artistic ideal was at the service of humanity as a whole, whilst upholding protoracist notions on the exclusive ability of an ethnic group to achieve such harmony. Many of these ideas were even embodied in his artwork and not just in published writings and speeches. Itten's own interest in oriental art and culture coexisted uncomfortably with these tendencies, leaving an ambivalent and problematic legacy, which has recently been pointed out by some scholars of the movement. Here we will analyze some aspects of this contradiction and its implications from the comparatist point of view. It is necessary to consider how these inconsistencies should be analyzed if we wish to avoid falling into a trivial and uncritical position regarding a cultural movement as relevant as the Bauhaus school.
\end{abstract}

Keywords: Bauhaus, Johannes Itten, Walter Gropius, occultism, Mazdaznan, comparatism 


\begin{abstract}
A
o largo de su historia, la Bauhaus fue más multifacética que monolítica. Si bien fue el impulso de Walter Gropius y su visión de la arquitectura y las artes lo que dio origen al movimiento, y si él fungió como su representante vitalicio aún años después de que la Bauhaus cerrara, sus sucesores, Hannes Meyer (1928 - 1930) y Mies van der Rohe (1930 - 1933), dieron cada quien una cara distinta al movimiento y enfatizaron las prioridades de la institución en función de intereses diversos. Pero hubo también tendencias dentro de la escuela que contrastaron casi hasta el antagonismo con la posición original de Gropius y que tuvieron una gran relevancia. Se trata, sobre todo, del pensamiento esotérico y orientalista de Johannes Itten y sus seguidores.

La visión de Gropius, que se mantuvo a lo largo de la historia de la escuela, tuvo como objetivo principal hacer de la Bauhaus una institución con aspiraciones académicas, formativas, al tiempo que reformadoras. En concordancia con los impulsos generalizados de la modernidad, Gropius deseaba que la escuela fuera pionera y representante del progreso. Desde el inicio, se dio a la tarea de reconciliar el arte y la técnica en disciplinas como la arquitectura. Así lo definió años después y desde el exilio en su libro sobre la Nueva Arquitectura y la Bauhaus, pues lo que buscaba era unir el valor práctico de la arquitectura con la "satisfacción estética del alma humana” (Gropius, 1965: 25). El camino no se presentaba fácil, pues se trataba de reconciliar dos perspectivas históricamente antagónicas. Y no sólo pretendía que la arquitectura representara esta unión, sino que las artes conciliaran y adoptaran la técnica y la tecnología dentro de su más profunda razón de ser. Como otros movimientos modernos, la visión no historicista y reformadora le permitió a la escuela adoptar y desarrollar propuestas de artistas que rompían con todo antecedente, como Wassily Kandinsky, Paul Klee y Johannes Itten, y esto la colocó en una posición privilegiada tanto para artistas consolidados como para estudiantes interesados en el arte.

Basta leer el Manifiesto Bauhaus, de 1919, para advertir tanto las principales propuestas de Gropius, como el trabajo en conjunto entre artistas y artesanos. Gropius culpaba a las academias de formar arquitectos y artistas desarmados para
\end{abstract}


la lucha de la vida. Desde su perspectiva, la formación de dichas academias era diletante, apartada de la realidad y con poca técnica y adiestramiento en el taller. La primera frase del documento es inequívoca al respecto: "Das Endziel aller bildnerischen Tätigkeit ist der Bau!" (Gropius, 1919:1). La meta final de toda actividad plástica es la construcción. Y mucho antes de su intervención en la Bauhaus, Gropius manifiesta en sus escritos tempranos una tendencia antimaterialista, señalada acertadamente por Joaquín Medina Warmburg (2018: 68) en su prólogo a la recopilación en español de los escritos y conferencias de Gropius. En su ensayo "Sobre la esencia de la distinta voluntad artística en Oriente y Occidente", fechado en 1910, Gropius señala los conceptos de Alois Riegl (Kunstwollen o voluntad artística) y la teoría de Gottfried Semper que comprende a la obra de arte como un producto mecánico de tres factores: la voluntad artística, el uso práctico, y los materiales y la técnica. En este mismo escrito Gropius afirma que "la obra de arte consumada depende de la feliz conjunción y del equilibrio entre dos extremos de la voluntad artística humana” (Medina Warmburg, 2018: 97), dejando clara una postura en la que buscaba siempre un equilibrio entre la técnica y el arte.

Formalmente, el movimiento existió entre 1919 y 1933, una época muy turbulenta en Alemania. La Bauhaus encarnó, tal vez injustamente, la enemistad entre una amplia corriente cultural conservadora y un espectro político de izquierdas, lo cual, ya hacia los años del Tercer Reich, le valió la caracterización de ser una institución "bolchevique", "internacionalista” y "judía”. Éstas fueron las razones citadas por el régimen nazi para su clausura definitiva. Casi todos los miembros de la Bauhaus emigraron, pero muchos de los integrantes judíos fueron asesinados.

\section{La literatura y el arte en la era industrial: Scheerbart y Taut como preludio}

Existe un vínculo poco explorado entre el escritor alemán Paul Scheerbart y las ideas dominantes acerca de la arquitectura en la Bauhaus. En su obra más conocida, Arquitectura de cristal, Scheerbart defendió, por ejemplo, la sustitución del ladrillo por el cristal. La teoría fue puesta en práctica por Bruno Taut en el Pabellón de Cristal de la Exposición de la Deutscher Werkbund de Colonia en 1914. 
A Scheerbart se le recuerda mejor a través de citas oscuras de Walter Benjamin, Walter Gropius y Bruno Taut. Aun en la era de la cultura trepidante de Berlín de aquel tiempo, Scheerbart fue un narrador, editor e inventor totalmente excepcional. Desde finales de la década de 188 o hasta su muerte prematura en 1915, escribió prolíficamente sobre ciencia, planificación y diseño urbano, viajes espaciales y políticas de género, a menudo en el curso de un solo texto.

Un año antes de morir, publicó su tratado más célebre, Glasarchitektur (Arquitectura de Cristal), en el que predijo una civilización superior tecnocrática, cuyo orden pacífico se originaría a partir de la proliferación de ciudades de cristal y continentes flotantes hechos de vidrio cromático, una visión resumida en su frase "La arquitectura de cristal enriquecerá a la humanidad en lo ético" (Scheerbart y Taut, 1972: 63). Es claro que las ideas de Scheerbart tienen una resonancia no sólo en el proyecto Bauhaus, sino en la mezcla de ideales tanto espirituales como materiales que caracterizaron a la escuela desde su fundación.

El momento de Scheerbart fue uno en el que la tecnología y el diseño tenían un potencial evocador que, aunque puede considerarse futurista, hunde sus raíces en el pasado. En contraste, las personas que se opusieron a él o ridiculizaron sus ideas, quienes más o menos ganaron la discusión después de la Primera Guerra Mundial, pensaron que la eliminación del pasado era esencial. Esta incorporación del pasado en un proyecto orientado hacia el futuro separa a Scheerbart de muchos movimientos modernistas.

Sin embargo, es posible apreciar en Gropius una clara afinidad con los ideales artísticos y técnicos de Scheerbart. En su escrito de 1910, Gropius escribe "sólo con la ayuda de medios ostensibles, materiales, podemos representar en el arte la idea espiritual, puesto que permanecemos encadenados al mundo terrenal de los fenómenos" (Medina Warmburg, 2018: 117). Tanto en Arquitectura de cristal, en su novela Lesabéndio y en su fascinante diario ficcional Das Perpetuum Mobile, Scheerbart explora los ideales conciliatorios entre el arte y la técnica de una forma muy similar a la "feliz conjunción" de la voluntad artística y la técnica que influyó a Gropius y que caracterizó desde sus inicios a la escuela. 
Los propósitos fundamentales de Johannes Itten cuando en 1919 se incorporó como profesor a la escuela fundada por Gropius se enfocaban en "liberar las fuerzas creativas en sus estudiantes" para que después pudieran integrarse a la industria como artistas (Itten, 1963: 8). De esta manera, Itten se inserta en el programa artístico y técnico de la escuela, pero como veremos a continuación, lo hace desde una ideología fuertemente comprometida con ciertas corrientes de esoterismo occidental.

\section{El "Curso Introductorio" de Johannes Itten y el Mazdaznan}

Entre las múltiples huellas que dejó la Bauhaus para diversas disciplinas está el llamado "Curso Introductorio", que en un inicio fue concebido en su totalidad por el artista suizo Johannes Itten. A su salida de la escuela, a Itten le sucedieron Josef Albers y Lazlo Moholny-Nagy como continuadores del programa, aunque cada uno adaptó e hizo modificaciones.

En 1919, el primer año de la escuela, Itten fue uno de los nueve artistas invitados a colaborar en ella. Gerhard Marcks, un escultor, llegó también en 1919. Y cada año fueron incorporándose pintores como Lyonel Feininger (quien se encargó de la imagen en el Manifiesto de 1919 de Gropius), George Muche en 1920, Oskar Schlemmer en 1921, Paul Klee en 1921, Lothar Schreyer en 1921, Wassili Kandinsky en 1922 y Lazlo Moholny-Nagy en 1923.

Durante esta etapa inicial de la escuela prevalecieron modelos esotéricos de vida que, gracias a Itten y a Muche, resultaron de gran influencia entre los estudiantes. Ambos artistas eran adeptos a la enseñanza Mazdaznan, una corriente o secta esotérica que se autonombraba heredera del zoroastrismo (Linse, 2017: 2). Tal fue la influencia de estas prácticas e ideología en la Bauhaus que en retrospectiva el mismo Gropius reconoció que la escuela no tenía un programa definido completamente: el formalismo abstracto y la producción en serie convivían con una predisposición a las tendencias esotéricas de diversa índole, como el Wandervogelbewegung, un movimiento de jóvenes que protestaban en contra de la industrialización, y el propio Mazdaznan o el yoga y el vegetarianismo (Hüttner y Leidenberger, 2019: 228). 
A partir del establecimiento de la Sociedad Teosófica en Alemania en 1884, proliferaron entre diversos círculos personas que se interesaron y se hicieron miembros activos de muchos grupos. En 1902, Rudolf Steiner se unió a la teosofía para años después fundar su propio movimiento, la antroposofía. Entre estas escuelas surgió el Mazdaznan, un movimiento menos popular que tenía muchas coincidencias con la teosofía en tanto que retomaba en una forma similar algunas enseñanzas y prácticas de origen oriental. Entre sus ideas principales estaba que, mediante la respiración, ciertos movimientos acompañados de una dieta estrictamente vegetariana y el pensamiento esotérico, era posible ascender a una conciencia superior.

El movimiento Mazdaznan tuvo su origen en la década de 1890 en Estados Unidos. Fue fundado por Otto Hanish, quien cambió su nombre a Dr. Otoman Zar-Adusht Hanish. Según Hanish, la libertad de pensamiento podía ser alcanzada con ejercicios de respiración y movimientos afines al yoga tomados del hinduismo. Hanish consideraba el Mazdaznan como el único camino verdadero, pero sus enseñanzas no tenían mucha claridad. Además de los movimientos, la dieta vegetariana y la respiración, otro aspecto importante eran las irrigaciones colónicas y la abstinencia sexual (Wedemeyer-Kolwe, 2004: 153).

Los vínculos entre el esoterismo y figuras conocidas en el ámbito artístico eran mucho más comunes de lo que podríamos imaginar. Alma Mahler, quien fuera esposa de Gropius durante esta época, era teosofista y fue ella quien recomendó a Itten. Dentro de la Bauhaus, tanto este último como Schreyer y Gertrud Grunow eran miembros de la secta Mazdaznan. Grunow, por su parte, era la única mujer docente en la Bauhaus de la época de Weimar y enseñaba teoría de la armonización musical. Ella también adoptó estas ideas sectarias en su pedagogía. Los tres dejaron la escuela en 1923, principalmente porque comenzaron a producirse conflictos entre los estudiantes interesados en las enseñanzas Mazdaznan y los otros, lo cual causó mucha preocupación a Gropius, quien anteriormente se había mostrado poco interesado en las peculiares prácticas aplicadas por Itten en toda la escuela.

A Gropius comenzó a preocuparle la intensidad con la que algunos profesores y estudiantes llevaban a cabo las prácticas ocultistas. En sus relatos de la 
época, algunos estudiantes advirtieron que las rupturas eran cada vez más evidentes (Linse, 2017: 8). Los "iniciados" rechazaban a los otros, por ejemplo, porque no tenían las mismas herramientas humanas y artísticas. Mientras tanto, Gropius quiso devolver a la escuela su enfoque original en la técnica y en una tendencia industrializadora que conciliara ideales artísticos al mismo tiempo, así que llamó a Paul Klee y a Oskar Schlemmer para crear un contrapeso a la gran influencia que Itten tenía en ese momento en la escuela.

A pesar de la salida definitiva de Itten de la Bauhaus, está claro que la influencia de Mazdaznan y del esoterismo fue significativa para el modelo educativo del curso introductorio. Éste siguió siendo usado en la escuela después de la salida de Itten, y sus fundamentos aún son vigentes en academias de arte en todo el mundo. Este curso es considerado hasta hoy una de las aportaciones académicas de la Bauhaus.

Así como las enseñanzas de la teosofía fundada por Madame Blavatsky se vieron envueltas en múltiples controversias, el Mazdaznan tenía creencias mucho menos inocuas. En particular, la reivindicación de los descendientes de Ainyahita, el pueblo ario. Ésta, como cualquier otra creencia en algún pueblo elegido, podía fácilmente convertirse en una filosofía de la superioridad racial. Así, desde 1907 la literatura Mazdaznan sirvió para propagar ideas racistas. El propósito principal de las prácticas era la creación de un "nuevo hombre", un nuevo tipo de raza superior con capacidades mentales más desarrolladas y con un cuerpo superior. Y esta nueva raza debía derivar directamente de la raza aria, considerada superior por Hanish (Linse, 2017: 7-8). Itten colaboró en revistas de la época y llegó a discutir abiertamente la supuesta superioridad de la raza aria. Una de sus pinturas, "La casa del hombre blanco" (1920) (Fig. 1), proporciona indicios de que Itten tenía coincidencias con las vertientes racistas del Mazdaznan. 
Figura 1

La casa del hombre blanco (1921-1924), Johannes Itten

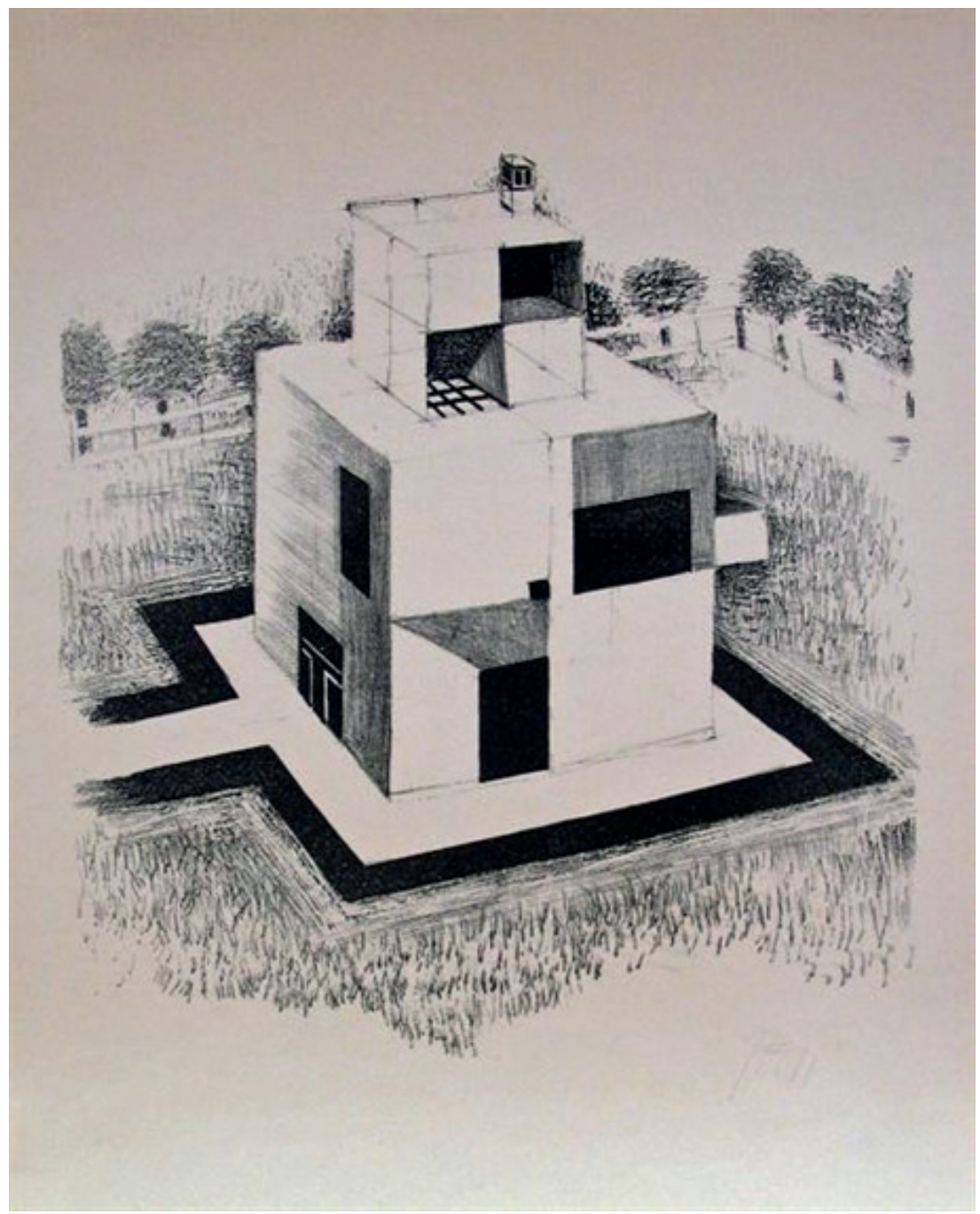

Nota: Litografía. Original en MoMA, Nueva York, Estados Unidos. Fuente: https://www.wikiart.org/ en/johannes-itten/haus-des-weissen-mannes-1924. Dominio Público.

Johannes Itten se relacionó con las enseñanzas Mazdaznan desde 1912. Hanish mismo visitó Alemania y Suiza en 1911, mientras que Itten fue miembro del templo Ario Mazdazdan en Herrilberg, cerca de Zürich. Esto se reflejó en sus obras, tal como en "Ascensión y pausa" (1919), en la que puede distinguirse el simbolismo perteneciente al movimiento. 
Las tendencias racistas del Mazdaznan pueden verse claramente en otros aspectos de su obra. En 1921 Itten pintó uno de sus cuadros más famosos, "Retrato de un niño" (Fig. 2), en el que representa a un infante (su hijo Mathias), vinculado con referencias astrológicas y una simbología de color que lo relaciona con las expectativas del futuro. Mathias (Buali, el nombre con el que Itten se refiere a su hijo) aparece como representante del nuevo ser humano (una especie de mesías) que debía ser de raza blanca, y los símbolos esotéricos en el cuadro brindaban también esos significados (Wagner, 2009).

\section{Figura 2}

Retrato de un niño (1921-1922), Johannes Itten

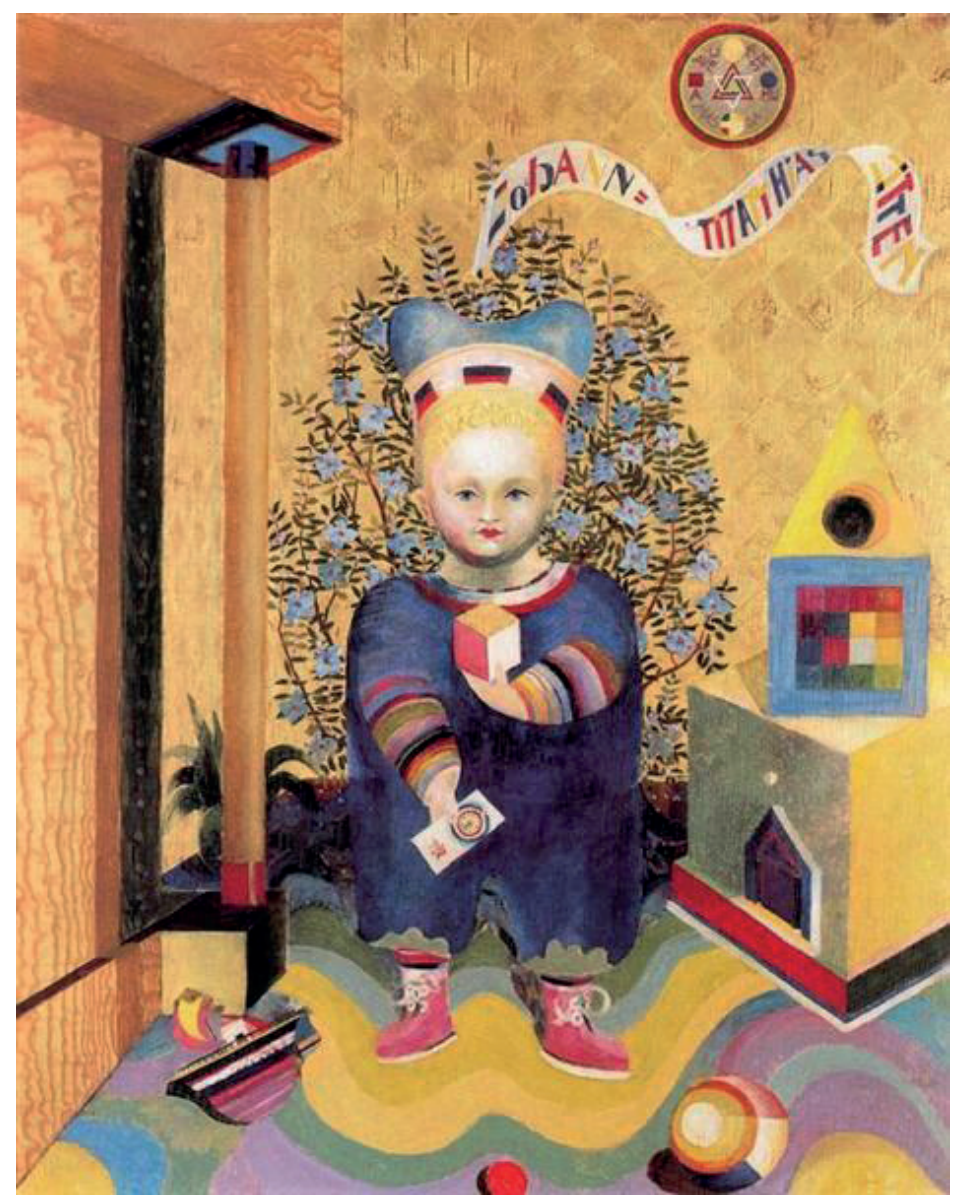

Nota: Óleo sobre tela. Original en Kunsthaus Zúrich, Suiza. Fuente: https://www.wikiart.org/en/ johannes-itten/children-s-portraits-1922. Dominio público. 
Las técnicas de respiraciones y movimientos que incluyó Itten en la formación de los estudiantes pretendían romper con la moral prevaleciente y producir un acercamiento sin mediaciones a un sentido estético. Itten (1963) describió su técnica como un triunfo sobre lo académico ortodoxo: "La educación del cuerpo como instrumento del espíritu es de gran importancia para los seres creativos” (12). Desde luego, en todo esto está también presente la influencia de Kandinsky, quien en Lo espiritual en el arte ya enfatizaba la idea de despertar la capacidad de develar lo espiritual a partir de lo material y lo abstracto. Kandisnky fue miembro de la Sociedad Teosófica desde 1909. En dicho texto le dedica algunas líneas a la teosofía y cita del libro de Madame Blavatsky, La clave de la teosofía (Goodrick-Clarke, 2008: 232).

Los profesores aliados al Mazdaznan, Grunow, Muche, Schreyer e Itten, siguieron desarrollando las ideas de lo que llamaron Lebensreform (reforma de vida) alemana, que por principio debía ser enemiga de la técnica y de la gran ciudad. Esta reforma de vida fue experimentada por los estudiantes seguidores del trío a través de las prácticas de respiración, la dieta vegetariana y la técnica de movimientos corporales. En contrapeso, Gropius insistió en el lugar original de la técnica en su proyecto y defendió su ideal de conciliar al ser humano con la técnica a través del arte.

Debe mencionarse que Itten nunca estuvo afiliado al partido Nacionalsocialista, pero Lothar Schreyer, su colega en la Bauhaus, sí. Schreyer fue uno de los 88 artistas que firmaron la carta llamada "Gelöbnis treuester Gefolgschaft" (que puede traducirse como "Voto de la alianza más fiel") firmada en 1933 y que sirvió como un voto de confianza público hacia Hitler desde el ámbito intelectual. A pesar de esto, los nazis clasificaron la obra de Schreyer como "arte degenerado", en la famosa exhibición de 1937. Era común en el nazismo que las aparentes alianzas fueran desechadas sin mayores miramientos cuando percibían una contradicción con sus espurias ideas. Algo similar sucedió más tarde entre el nazismo y el ocultismo, a pesar de que en sus inicios existieron lazos concretos, como la relación entre Hitler y Hanussen, por ejemplo. Desde 1938 - 1939 los nazis emprendieron una campaña abierta en contra de todos los grupos ocultistas, incluyendo los Mazdaznan desde 1933 (Conway, 2001: 371). 
Después de la salida de Itten, el artista húngaro Lazlo Moholny-Nagy continuó impartiendo el "Curso Introductorio", pero con un enfoque más conciliatorio con los objetivos de Gropius. En la concepción artística de Moholny-Nagy cabía la asociación de ideas biológico naturalistas con lo técnico y progresista. Creía en la liberación del ser humano a través de una práctica artística en el mundo técnico. A esto responden sus contribuciones más significativas en el arte, como su concepto de la.Neues Sehen (Nueva Visión), que propone que la cámara puede crear una visión totalmente nueva del mundo, inalcanzable para el ojo humano.

\section{Itten y la ceremonia del té}

En un artículo reciente, la académica Helena Čapková (2017) explora otro de los motivos, mucho más auténticamente orientales esta vez, que fueron importantes para Itten después de su salida de la Bauhaus. Bruno Taut, uno de los arquitectos que más influencia tuvo en Gropius y en la Bauhaus, había comenzado a tender puentes con artistas y arquitectos en Japón desde su visita a ese país en 1932. En sus conferencias tituladas "Fundamentos de la arquitectura japonesa", Taut afirmaba curiosamente que lo exótico de Japón ya no existe en Europa y que lo exótico de Europa ya no existe en Japón. En otras palabras, para Taut, Japón ya no es un símbolo de lo exótico porque la cercanía o la hibridación (para usar el término de Homi Bhabha) ${ }^{1}$ con Europa era ya una realidad. Tal vez hay una cierta exageración en lo que dice Taut, pero hay que tomar en cuenta que ya desde hacía varios años existía una comunicación con el arte japonés. El término "japonismo" se usó por primera vez en 1872 .

Itten se había interesado por las filosofías japonesa y china. Como mencioné anteriormente, se sumergió en la ola de esoterismo que llenó Alemania desde las primeras décadas del siglo XX, durante la cual las enseñanzas de Meister Eckhart

${ }^{1}$ Uso el témino aquí en el sentido más amplio, como una especie de mezcla de culturas del Oriente y Occidente. En DissemiNation: Time, Narrative, and the Margins of the Modern Nation, Bhabha habla de sujetos poscoloniales por excelencia que desdibujan las fronteras de lo nacional. Al respecto, coincido con la crítica que hacen de este concepto Mishra y Hodge (2005: 383). 
y Jakob Böhme cobraron relevancia, junto con las de Budha y Lao Tsé. Al igual que los motivos ocultistas del Mazdaznan, las filosofías orientales fueron importantes para determinar los objetivos de su "Curso Introductorio". Itten pretendía que en éste se desarrollaran habilidades creativas y un potencial artístico libre de convenciones. Concretamente, a partir del modelo del arte tradicional japonés, al explicar el ritmo de los pinceles, las líneas y la composición, Itten utilizaba ejemplos de pinturas de tinta japonesa y china. Hacía que sus estudiantes pintaran sensaciones en unidad con el objeto que se pintaba y no en mimesis. Los hacía dibujar tanto sentimientos abstractos como fenómenos observables como la lluvia o la primavera. Hoy en día es difícil encontrar un curso sobre arte en cualquier nivel educativo que no contenga algunas de estas prácticas.

Pero entre los elementos orientales que influyeron a Itten, uno de gran relevancia fue la ceremonia del té y la filosofía en torno a ella: el téismo. Un documento fundamental por su difusión en Europa fue The Book of Tea (1906) de Okakura Kakuzo. Se trata de una obra escrita originalmente en inglés y pensada para el público europeo. Describe la historia del téismo en Japón a partir del siglo XV. Esta filosofía es descrita como un "culto que se basa en la adoración de lo bello entre los aspectos sórdidos de la existencia cotidiana” (Kakuzo, 2016: 29). También se describe como "esencialmente una adoración de lo imperfecto, como una forma de lograr algo posible dentro de esta cosa imposible que llamamos vida” (Kakuzo, 2016: 34).

Se trata además de un documento importante para las teorías postcolonialistas, pues hace referencia directa, en un tono sarcástico y humorístico, a los prejuicios abundantes entre la cultura occidental y la oriental. El autor se pregunta desde el principio del ensayo qué puede estar mal con el hecho de que los occidentales se diviertan con "nosotros", si al fin y al cabo lo que sabíamos del Occidente hasta hace no mucho consistía en que predican lo que no hacen, tal como muchos de los misioneros (Kakuzo, 2016: 11).

El autor expone también que el téismo tiene sus raíces en el taoísmo y el zen. Según nos dice, al menos en Japón y en la antigua China, el té es para los iniciados una forma de estar en contacto con las filosofías de Confucio, Lao Tsé y 
hasta Sakyamuni. Sin embargo, hace una clara distinción entre ellos, favoreciendo la visión taoísta, concretamente la de Lao Tsé y Zhuanzi, ya que ésta trata de encontrar la belleza en este mundo de calamidad y preocupación. Este enfoque, que podríamos llamar materialista en algún girado, es lo que acerca la ceremonia del té al arte. Desde esta perspectiva, lo mundano tiene el mismo valor que lo espiritual. El taoísmo, comenta el autor, estableció los fundamentos para los ideales estéticos y el téismo los volvió prácticos y aplicables a la realidad. Todo esto debe expresarse en la realización concreta de la ceremonia del té. Por ejemplo, si se está usando una tetera redonda, comenta el autor, la jarra debe ser angular. El sentido de este arreglo es que no debe haber repetición o monotonía en la estética del lugar.

El libro incluye un capítulo acerca del arte y su lugar en la filosofía del té. Para ilustrar esto, Kakuzo utiliza otro relato de la tradición taoísta en el que un monje que era conocido como un gran músico explica que su secreto es permitir que el arpa elija su tema. Al ejecutar su instrumento pierde la noción de si el arpa es él o él es el arpa. Éste es el secreto que posee el arte. Esta fusión con el objeto de la creación artística es también lo que Itten pretendía lograr a través de las técnicas y los ejercicios en su "Curso Introductorio". Es tal la importancia del arte para el téismo que en algún momento la coloca por encima de la religión: "en la religión el futuro está detrás de nosotros, mientras que en el arte, el presente es eterno" (Kakuzo, 2016: 62). Este sentido de lo eternamente presente se lograba con la ceremonia del té, que era también una forma del arte.

Los estudiantes de intercambio japoneses Iwao y Michiko Yamawaki llegaron a la escuela Bauhaus en 1930, durante el periodo en el que la academia se localizó en la ciudad de Dessau. Michiko fue alumna en el taller de textiles, mientras que su esposo Iwao se convirtió en uno de los fotógrafos de arquitectura más destacados de la escuela. Michiko publicó un libro llamado la "Bauhaus y la ceremonia del té” en el que explora las líneas de contacto entre la escuela y el téismo (Čapková, 2017: 108). Iwao fue el autor del famoso collage "El ataque a la Bauhaus" (1932), en el que ilustra la forma en que el gobierno nazi identificó la escuela con una institución bolchevique y anti-germana. Para este momento, Itten había dejado la escuela, pero su influencia en el Vorkurs seguía presente. 
Tras su rotundo triunfo electoral en 1932, los nacionalsocialistas dominaron la discusión político-cultural. Tratando de contener el daño a la Bauhaus, no sólo Gropius, sino también el director del museo, Ludwig Grote, el alcalde Hesse y críticos como Bruno E. Werner, pretendieron demostrar que las corrientes modernas, entre las cuales se contaba la escuela, no eran culturalmente bolcheviques, sino "alemanas". Por supuesto, nada de lo que argumentaron cambió la opinión de los oficiales nazis.

Años más tarde, en 1955, Itten volvió a encontrar a Gropius en la inauguración de la Hochschule für Gestaltung en Ulm. Después de sus viajes por Japón, India y Myanmar, Itten estaba convencido de que las culturas orientales y occidentales debían relacionarse más. Ya desde 1932, la escuela de arte de Berlín fundada por Itten recibió al pintor japonés Takehisa Yumeji para enseñar la técnica del pincel. Este interés por el arte y la cultura oriental parecía estar en contradicción con las visiones racistas del Mazdaznan, pero a la vez funcionaban como un "contrapeso" (Linse, 2017: 8), el cual no deja de ser, en apariencia al menos, irreconciliable.

\section{Literatura comparada en los estudios culturales}

En años recientes, la disciplina de la literatura comparada ha adoptado cada vez más una postura afín a la teoría postcolonialista, en el sentido en que se estudian culturas en su relación mutua. Es importante notar, como sabemos, que un acercamiento transnacional a la cultura no siempre resulta en el desdibujamiento de las fronteras ni en el rescate de las minorías. Muchas de las corrientes ocultistas de finales del siglo XIX y principios del siglo XX terminaron por ser afines a filosofías supremacistas cuyas consecuencias fueron terribles.

Irónicamente, durante el nacionalsocialismo en Alemania, el ministro de propaganda llevó a cabo una política cultural supuestamente internacionalista de la cual se habla muy poco hoy en día (Lubrich, 2006: 52). Con la intención de dominar la actividad cultural europea desde Alemania, el partido en el poder organizó congresos y encuentros en Weimar en 1941 y 1942, en los que participaron autores de todo el continente. Entre sus actividades estaba la producción de una revista 
académica de literatura europea en la que se publicaban ensayos, poemas, traducciones y artículos sobre teoría literaria y reseñas sobre obras de autores de distintos países.

Con su genuino interés en las culturas orientales, así como con la adopción de prácticas de yoga y vegetarianismo, Itten quedó envuelto en una paradoja similar a la que hoy es evidente en el proyecto cultural internacionalista del nazismo que señala Oliver Lubrich. El trasfondo racista que no puede separarse de su obra deja en evidencia las profundas contradicciones culturales que no son compatibles con su afán por mirar hacia el Oriente.

La literatura comparada debe ser consciente de que una visión postcolonialista carente de crítica y de realidad histórica puede resultar en una trivialización transnacionalista. De ahí que resulte necesario señalar las contradicciones presentes en figuras como Itten, Schreyer y en la Bauhaus en general. Está claro que la comparatística puede fácilmente caer en esta trivialización aun cuando su intención sea distinta. Estas preguntas siguen siendo válidas en el estudio de la cultura y la literatura a pesar de que tenemos muy presente el peligro que acecha en el acercamiento desde una cultura a otra.

\section{REFERENCIAS BIBLIOGRÁFICAS}

ČAPKOvÁ, Helena. (2017). "Bauhaus and Tea Ceremony: A Study of Mutual Impact in Design Education between Germany and Japan in the Interwar Period". In Carolien Stolte y Yoshiyuki Kikuchi (Eds.), Eurasian Encounters: Museums, Missions, Modernities (pp. 103-120). Amsterdam University Press.

CONWAY, J.S. (2001). The Nazi Persecution of the Churches, 1933-1945. Regent College Publishing.

GOODRICK-ClARKE, Nicholas. (2008). The Western Esoteric Traditions: A Historical Introduction. Oxford University Press. 
GROPIUS, Walter. (1919). Bauhaus Manifest. Recuperado el 27 de febrero de 2021 de http://files8.webydo.com/94/9495476/UploadedFiles/AE227154-F565-F891A285-22D292573D04.pdf

GROPIUS, Walter. (1965). The New Architecture and the Bauhaus (P. Morton Shand, Trad.). The MIT Press.

HÜTTNER, Bernd; LEIDENBERGER, Georg (Eds.). (2019). 100 Fahre Bauhaus. Vielfalt, Konflikt und Wirkung. Metropol Verlag.

ITTEN, Johannes. (1963). Mein Vorkurs am Bauhaus, Gestaltungs- und Formenlehre. Otto Maier Verlag.

KAKUZO, Okakura. (2016). The Book of Tea. Benjamin Press.

LINSE, Ulrich. (2017). “Die Mazdaznan-Pädagogik des Bauhaus-Meisters Johannes Itten”. En Peter Bernhard (Ed.), Bauhaus Vorträge. Gastredner am Weimarer Bauhaus 1919-1925. Mann Verlag.

LUBRICH, Oliver. (2006), "Comparative Literature - in, from and beyond Germany". Comparative Critical Studies, 3(1-2), 47-67.https://doi.org/10.1353/ccs.2006.0012 Medina Warmburg, Joaquín (Ed.). (2018). Walter Gropius. Proclamas de Modernidad. Reverte.

MiSHrA, Vijay; Hodge, Bob. (2005). “What Was Postcolonialism?”. New Literary History, 36(3), 375-402. https://doi.org/10.1353/nlh.2005.0045

SGHEERBART, Paul; TAUT, Bruno. (1972). Glass Architecture. November Books.

WAGNER, Christoph. (2009). Esoterik am Bauhaus: Eine Revision der Moderne? Schnell \& Steiner GmbH.

WEDEMEYER-KOLWE, Bernd. (2004). Der neue Mensch: Körperkultur im Kaiserreich und in der Weimarer Republik. Königshausen \& Neumann. 